\title{
Relación entre depresión, estrés y estresores en adolescentes gestantes bajo distintas condiciones de estado civil
}

\section{Relationship between depression, stress and stressors in pregnant teenagers under different marital status conditions}

\author{
Lucía del Carmen Quezada Berumen Mónica Teresa González Ramírez ${ }^{1}$ \\ Universidad Autónoma de Nuevo León, México
}

(Rec: Junio 2011 - Acep: Octubre 2011)

\begin{abstract}
Resumen
Los estudios e investigaciones que abordan la depresión y el estrés han tomado importancia dada su alta pertinencia, especialmente en el embarazo adolescente. Este estudio tuvo como propósito identificar la relación entre los niveles de depresión y estrés, así como el número de estresores enfrentados por 82 adolescentes primigestas, quienes respondieron a los instrumentos de evaluación: Inventario de Depresión de Beck, la Escala de Estrés Percibido y el Cuestionario de Sucesos Vitales. De acuerdo a los resultados, aquellas adolescentes que viven con la propia familia son quienes poseen las medias más altas de estrés y depresión, por lo que puede concluirse que el hecho de que dichas adolescentes vivan con la propia familia, podría ser un factor que contribuya con la depresión y el estrés durante el embarazo.
\end{abstract}

Palabras clave: Depresión, estrés, embarazo adolescente, condición de estado civil.

\begin{abstract}
Research on depression and stress has become important because of its high relevance especially in teenage pregnancy. This study aimed to identify the relationship between depression and stress levels and number of stressors faced by 82 first-time pregnant teenagers, who responded to the assessment instruments: Beck Depression Inventory, Perceived Stress Scale and Life Events Questionnaire. According to the results, adolescents who live with their own family are those with the highest means of stress and depression. Therefore, it can be concluded that adolescents who live with their own family are more prone to develop depression and stress during pregnancy, since this could be a contributing factor.
\end{abstract}

Key words: Depression, stress, teen pregnancy, marital status condition.

\footnotetext{
1 Correspondencia a: Mónica Teresa González Ramírez. Dirección: Facultad de Psicología, Universidad Autónoma de Nueva León. Dr. Carlos Canseco 110. Col. Mitras Centro. C. P. 64460. Monterrey, Nueva León, México. Tel: (52-81) 83338233. E-mail: monygzz77@yahoo.com, monica.gonzalezrz@uanl.edu.mx.
} 


\section{Introducción}

El embarazo en la adolescencia puede llevar a situaciones de riesgo tanto para la joven como para su hijo. La hipertensión en la joven (debido al embarazo y a la pobre asimilación nutricional que acarrea desnutrición en el recién nacido), dificultad para ganar peso en la madre como en el producto en gestación y bajo peso al nacer, así como el trabajo de parto espontáneo debido a rotura prematura de las membranas ovulares, restricción del crecimiento intrauterino, amenazas de aborto y hemorragias, son algunos de los riesgos más frecuentes en el embarazo adolescente (Díaz-Franco, 2007; Oviedo, Lira, Ito \& Grosso, 2007).

Ser madre adolescente o estar embarazada en la adolescencia es considerado como un gran factor de riesgo para el producto en gestación (Ramos, Barriga \& Pérez, 2009). Se destaca el nacimiento prematuro y su bajo peso, la pobre absorción de nutrientes y desnutrición en edad gestacional. Datos de América Latina, incluyendo México, revelan que los riesgos en el embarazo experimentados por adolescentes como el nacimiento prematuro, crecimiento fetal por debajo de los límites normales y la muerte neonatal, son mayores en esta población que en la de Estados Unidos de Norteamérica (Conde-Agudelo, Belizan \& Lammers, 2005).

La depresión es una de las situaciones de riesgo que unida a otras condiciones puede afectar el embarazo y al recién nacido con consecuencias lamentables, como lo concluye el estudio de Sepúlveda, Bustreo, Tapia, Rivera, Lozano, Oláiz, Partida, García-García y Valdespino (2006), donde el impacto negativo en los niños cuya madre padecía depresión en el embarazo, incluye el bajo peso al nacer, crecimiento incompleto, episodios de diarrea y una inmunización incompleta y desnutrición. El riesgo de padecer depresión en la mujer se incrementa con el embarazo, ya que se considera un periodo crítico del desarrollo psicológico y que pone a prueba el nivel de madurez emocional de la mujer así como su propia identidad (Gómez \& Aldana, 2007).

Apuntando en la misma dirección, es sabido que la depresión comúnmente coexiste con otros desórdenes, particularmente en niños y adolescentes (Joshi, 1998). De acuerdo a Bartolomucci y Leopardi (2009), el vínculo entre estrés y depresión no es nuevo, ya que la noción de que el estrés puede causar depresión ha sido un concepto subyacente. Lazarus (2000) considera que el estrés es interdependiente de las emociones (depresión). Asimismo, Sánchez, Garrido y Álvaro (2003) afirman que el estrés se incluye en prácticamente todos los modelos teóricos existentes sobre el deterioro psicológico.
El estrés es derivado de eventos estresantes, particularmente estresores crónicos asociados con bajo status socio-económico (considerado por Sandín, 1999, como una variable moduladora del estrés), así como la baja satisfacción con el apoyo social y los problemas con la pareja, que a su vez son ligados como predictores de depresión durante el curso del embarazo (Pajulo, Savonlahti, Souraner, Helenius \& Piha, 2001, O'Hara, 1994, en Grote \& Bledsoe, 2007; Ritter, Hobfoll, Lavin, Cameron \& Hulsizer, 2000, en Zelkowitz, Schinazi, Katofsky, Saucier, Valenzuela, Westreich \& Dayan, 2004; Zuckerman, Amaro, Bauchner \& Cabral, 1990).

El embarazo es un periodo de cambios significativos para la mujer y sus acompañantes (Billingham, 2011) y como principales fuentes de estrés se incluyen el cambio de roles, cambio de vida y dificultades en las relaciones (Ramsey, Abell y Baker, 1986). Un número considerable de estudios asocian el estrés experimentado en el embarazo con el bajo peso, tanto en el recién nacido como en su edad gestacional, así como el nacimiento pretérmino (Latendresse, 2009, Stanton, Lobel, Sears y Del Luca, 2002, en Alderdice y Lynn, 2009).

Hoy en día se cuentan con numerosos estudios acerca del embarazo adolescente asociado a la depresión y sus consecuencias (Da Costa, Dritsa, Larouche \& Brender, 2000; Figueiredo, Bifulco, Pacheco, Costa \& Magarinho, 2006; Ramos et al., 2009; Sepúlveda et al., 2006) así como a algunas variables sociodemográficas, como el estado civil. Estos estudios revelan que las adolescentes embarazadas casadas o cohabitando muestran menos niveles de depresión a diferencia de las solteras (Deal \& Holt, 1988; Figueiredo et al., 2006).

De acuerdo a Díaz (2003), director general de Mexfam, los adolescentes no son un grupo homogéneo, sus expectativas y necesidades varían de manera importante por razones de edad, sexo, estado civil y clase social, por lo que el embarazo en esta edad supone un fuerte obstáculo para el cumplimiento de metas y expectativas en la vida futura.

Como anteriormente se revisó, el hecho de tener un embarazo en la adolescencia acarrea una serie de riesgos que ponen en juego el desarrollo integral, tanto de la madre como del producto. Para Kovacs (2006), la depresión es un padecimiento común entre los adolescentes, y aún más en las adolescentes embarazadas siguiendo a Whitman, Borkowski, Keogh y Weed (2001, en Lanzi, Bert, \& Jacobs, 2009). Vinculado a variables como el estado civil, ésta se manifiesta en diferentes niveles, lo cual conlleva serias repercusiones.

Por lo anterior, se considera de gran importancia ahondar en factores que aporten información sobre 
Tabla 1. Descriptivos de edad, escolaridad y meses de embarazo por condición de estado civil.

\begin{tabular}{ccccc}
\hline Condición de estado civil & & Edad & Escolaridad & $\begin{array}{c}\text { Meses de } \\
\text { Embarazo }\end{array}$ \\
\hline \multirow{3}{*}{ Soltera viviendo con la propia familia } & Media & 17.23 & 10.55 & 4.05 \\
& Mediana & 17.00 & 11.00 & 4.00 \\
& D.E & 1.023 & 1.434 & 1.36 \\
Casada/unida viviendo & Media & 18.00 & 11.12 & 5.00 \\
con la propia familia & Mediana & 18.00 & 12.00 & 5.00 \\
& D.E & 1.061 & 1.536 & 1.82 \\
Casada/unida viviendo & Media & 17.35 & 10.65 & 4.51 \\
con la familia de la pareja & Mediana & 17.00 & 11.00 & 4.00 \\
& D.E & 1.152 & 2.228 & 1.58 \\
Pareja viviendo sola & Media & 18.82 & 12.55 & 5.09 \\
& Mediana & 19.00 & 13.00 & 5.00 \\
\hline
\end{tabular}

aquello que pudiera contribuir o facilitar la depresión en las adolescentes gestantes, como lo es su estado civil y la familia de residencia de las jóvenes.

Es por ello que el presente estudio pretende identificar la relación entre el estrés, el número de estresores y los niveles de depresión en adolescentes primigestas divididas en cuatro grupos denominados condiciones de estado civil: solteras viviendo con la propia familia; casadas/unidas viviendo con la propia familia; casadas/unidas viviendo con la familia de la pareja y la pareja viviendo sola (casadas/unidas que viven independientemente de los senos familiares).

\section{Método}

\section{Participantes}

La muestra estuvo constituida por 82 adolescentes primigestas con edades entre los 15 y 19 años pertenecientes a diferentes instituciones educativas de nivel medio, medio superior y superior, así como a instituciones públicas de salud de los municipios de Guadalupe y Zacatecas en el Estado de Zacatecas, México.

\section{Instrumentos}

Los instrumentos de evaluación utilizados para la realización de este estudio fueron:

- Inventario de Depresión de Beck (BDI), de Beck, Ward, Mendelson, Mock y Erbaugh (1961), en un formato de autoaplicación simplificado por Moral (2010) para medir la sintomatología depresiva.

- La Escala de Estrés Percibido de Cohen -PSS- (Cohen, Kamarak \& Mermelstein, 1983), en su versión para México de González y Landero (2007).

- $\quad$ El Cuestionario de Sucesos Vitales (CSV) de Sandín y Chorot (1987), para la evaluación de situaciones amenazantes (estresores).

En el análisis estadístico de los datos se utilizó el análisis de la varianza (ANOVA) de un factor para evaluar las diferencias entre los niveles de depresión, estrés y número de estresores entre los 4 grupos correspondientes a las condiciones del estado civil.

\section{Resultados}

La distribución del número de adolescentes para cada uno de los grupos que corresponden a las condiciones de estado civil, quedaron de la siguiente manera: Solteras viviendo con la propia familia: 31 , para las casadas/unidas viviendo con la propia familia: 17 , casadas/unidas viviendo con la familia de la pareja: 23 y para la pareja viviendo sola: 11 .

La edad promedio del total de la muestra fue de 17.63 años, 10.9 para los años de escolaridad y 4.5 para los meses de embarazo. En la tabla 1 se muestran los promedios de edad, escolaridad y meses de embarazo para las cuatro condiciones de estado civil. 
La descripción de los puntajes obtenidos en depresión, estrés y número de estresores reportados por las 82 adolescentes, se muestran en la tabla 2.

Tabla 2. Análisis descriptivo general.

\begin{tabular}{lccc}
\hline & Depresión & Estrés & Estresores \\
\hline $\mathrm{N}$ & 82 & 82 & 82 \\
Media & 36.48 & 26.37 & 15.02 \\
Mediana & 34 & 27.5 & 14.00 \\
Desviación estándar & 9.18 & 8.95 & 9.00 \\
\hline
\end{tabular}

Moral (2011) indica que para definir un caso de depresión, se podría adoptar como punto de corte aquellas puntuaciones mayores o iguales a 20 como lo proponen Vázquez y Sanz (1997) en España, sin embargo, los resultados obtenidos por Moral (2011), mostraron una media de 11.36 con una mediana de 9 ; los puntajes obtenidos en esta muestra son muy superiores.

Moral, González y Landero (en prensa) sugieren para la escala de estrés percibido un punto de corte de 30 o más para considerar casos de alto nivel de estrés, ya que éste queda justo por encima del promedio de las madres de pacientes oncológicos pediátricos (Moral \& Martínez, 2009), coincide con el promedio de mujeres con fibromialgia (González, García-Campayo \& Landero, 2011) y se ubica una desviación estándar de la media de población no clínica (Cohen \& Wiliamson, 1988; González \& Landero, 2007a).

Con el anterior panorama, se presentan en la tabla 3 los resultados correspondientes al objetivo fundamental de este estudio.
En la variable estresores y basándonos en sus 11 sub-escalas (Tabla 4), en las cuales se consideraron únicamente el número de estresores percibidos como negativos, el número de estos fue mayor en las solteras viviendo con la propia familia en relación a los otros grupos en las sub-escalas: trabajo, amor, hijos, social, residencia y académico. De la misma manera, para las sub-escalas de salud, pareja, familia, legal y finanzas, las casadas/unidas viviendo con la familia de la pareja obtuvieron el mayor número, seguido del grupo de las solteras viviendo con la propia familia. El grupo de la pareja viviendo sola reportó el menor número de estresores en todas las sub-escalas.

Cabe destacar que para este estudio la sub-escala de hijos está compuesta por un solo ítem, pues se consideró como el único ítem apropiado para la muestra (ya que este refiere al hecho de haber quedado embarazada), por lo que es importante señalar que dentro de las 31 solteras viviendo con la propia familia, para 20 de ellas fue considerado como un hecho negativo, mientras que para las 17 casadas/unidas viviendo con la propia familia y 23 casadas/unidas viviendo con la familia de la pareja, únicamente para 4 adolescentes de ambos grupos resultó ser un hecho negativo. Para el grupo de la pareja viviendo sola, compuesto por 11 sujetos, el haber quedado embarazada resultó ser un hecho negativo solo para dos de ellas.

\section{Análisis de comparación de grupos}

Para establecer si las medias de depresión, estrés y número de estresores presentan diferencias

Tabla 3. Descriptivos de depresión, estrés y número de estresores por condición de estado civil.

\begin{tabular}{llccc}
\hline Condición de estado civil & & Depresión & Estrés & Estresores \\
\hline Soltera viviendo con la & Media & 38.96 & 31.00 & 15.35 \\
propia familia & Mediana & 37.00 & 30.00 & 16.00 \\
& D.E & 8.10 & 7.06 & 6.09 \\
Casada/unida viviendo con & Media & 36.64 & 26.94 & 13.41 \\
la propia familia & Mediana & 35.00 & 30.00 & 10.00 \\
& D.E & 7.65 & 7.18 & 9.37 \\
Casada/unida viviendo con & Media & 35.91 & 23.43 & 17.65 \\
la familia de la pareja & Mediana & 31.00 & 22.00 & 16.00 \\
& D.E & 10.51 & 9.30 & 11.45 \\
Pareja viviendo sola & Media & 30.45 & 18.63 & 11.09 \\
& Mediana & 28.00 & 16.00 & 8.00 \\
& D.E & 9.48 & 8.59 & 9.22 \\
\hline
\end{tabular}


Tabla 4. Número de estresores negativos percibidos en las diferentes sub escalas del CSV por condición de estado civil.

\begin{tabular}{lcccc}
\hline Sub-escalas CSV & $\begin{array}{l}\text { Soltera viviendo con la } \\
\text { propia familia }\end{array}$ & $\begin{array}{l}\text { Casada/unida viviendo } \\
\text { con la propia familia }\end{array}$ & $\begin{array}{l}\text { Casada/unida viviendo } \\
\text { con la familia de la pareja }\end{array}$ & $\begin{array}{l}\text { Pareja viviendo } \\
\text { sola }\end{array}$ \\
\hline Trabajo & 24 & 8 & 20 & 4 \\
Salud & 7 & 5 & 10 & 1 \\
Amor & 20 & 11 & 9 & 3 \\
Pareja & 16 & 15 & 21 & 12 \\
Familia & 26 & 16 & 37 & 11 \\
Hijos & 20 & 4 & 4 & 2 \\
Social & 58 & 24 & 39 & 11 \\
Legal & 11 & 7 & 12 & 5 \\
Finanzas & 12 & 5 & 20 & 6 \\
Residencia & 21 & 7 & 20 & 5 \\
Académico & 23 & 12 & 11 & 1 \\
\multicolumn{1}{r}{ Total } & 238 & 114 & 203 & 61 \\
\hline
\end{tabular}

Tabla 5. Diferencias de grupo para las variables depresión, estrés y estresores.

\begin{tabular}{|c|c|c|c|c|c|c|}
\hline \multirow[b]{2}{*}{ Variable } & \multirow[b]{2}{*}{ Condición de estado civil } & \multirow[b]{2}{*}{$\mathrm{N}$} & \multirow[b]{2}{*}{ Media } & \multirow{2}{*}{$\begin{array}{l}\text { Desviación } \\
\text { Estándar }\end{array}$} & \multicolumn{2}{|c|}{ ANOVA } \\
\hline & & & & & $\mathrm{F}$ & $\mathrm{p}$ \\
\hline \multirow[t]{4}{*}{ Depresión } & Soltera viviendo con la propia familia & 31 & 38.97 & 8.10 & 2.497 & 0.66 \\
\hline & $\begin{array}{l}\text { Casada/unida viviendo con la propia } \\
\text { familia }\end{array}$ & 17 & 36.65 & 7.66 & & \\
\hline & $\begin{array}{l}\text { Casada/unida viviendo con la familia } \\
\text { de la pareja }\end{array}$ & 23 & 35.91 & 10.52 & & \\
\hline & Pareja sola & 11 & 30.45 & 9.48 & & \\
\hline \multirow[t]{4}{*}{ Estrés } & Soltera viviendo con la propia familia & 31 & 31.00 & 7.07 & 7.985 & .000 \\
\hline & $\begin{array}{l}\text { Casada/unida viviendo con la propia } \\
\text { familia }\end{array}$ & 17 & 26.94 & 7.19 & & \\
\hline & $\begin{array}{l}\text { Casada/unida viviendo con la familia } \\
\text { de la pareja }\end{array}$ & 23 & 23.43 & 9.31 & & \\
\hline & Pareja sola & 11 & 18.64 & 8.59 & & \\
\hline \multirow[t]{4}{*}{ Estresores } & Soltera viviendo con la propia familia & 31 & 15.35 & 6.10 & 1.565 & .205 \\
\hline & $\begin{array}{l}\text { Casada/unida viviendo con la propia } \\
\text { familia }\end{array}$ & 17 & 13.41 & 9.37 & & \\
\hline & $\begin{array}{l}\text { Casada/unida viviendo con la familia } \\
\text { de la pareja }\end{array}$ & 23 & 17.65 & 11.45 & & \\
\hline & Pareja sola & 11 & 11.09 & 9.22 & & \\
\hline
\end{tabular}


significativas entre las cuatro condiciones de estado civil de las adolescentes, se realizó el análisis de varianza (ANOVA) de un factor. Al contrastar la igual de varianzas de depresión, estrés y número de estresores mediante el estadístico de Levene, éste resultó no significativo para las variables de depresión y estrés $(p>.05)$ y significativo para la variable de estresores $(\mathrm{p}<.05)$.

\section{Resultados de ANOVA}

Los estadísticos de la prueba ANOVA de un factor (Tabla 5), permiten observar diferencias significativas entre los grupos de la variable estrés $(\mathrm{F}=7.985 ; \mathrm{p}=$ .001 ), por lo que se procedió a realizar el análisis posthoc con la prueba de Bonferroni para dicha variable. Por su parte, la variable depresión no mostró tener diferencias significativas entre los grupos $(\mathrm{F}=2.49$; $\mathrm{p}=.066$ ), motivo por el cual no se realizó el análisis post-hoc. De la misma manera, la variable estresores no presentó diferencia entre las medias de los grupos $(\mathrm{F}=1.565 ; \mathrm{p}=.205)$.

De acuerdo al análisis post-hoc con la prueba de Bonferroni, solo para la variable de estrés se observan diferencias significativas entre las medias de las condiciones de estado civil: soltera viviendo con la propia familia con la condición de casada/unida viviendo con la familia de la pareja $(\mathrm{p}=.006)$ y con la pareja viviendo sola $(\mathrm{p}=.001)($ Tabla 6$)$.

\section{Discusión}

De acuerdo a los resultados obtenidos en este estudio, se observa la presencia de casos de depresión y estrés para las cuatro condiciones de estado civil.

Dicho lo anterior, Whitman y otros (2001, en Lanzi y otros, 2009) argumentan que la depresión en adolescentes embarazadas se manifiesta en distintos niveles de acuerdo al estado civil, argumento que es compartido en este estudio, donde resultados similares a otros trabajos de investigación (Deal \& Holt, 1988; Figueiredo eta al., 2006) fueron los obtenidos. En dichos trabajos, los grupos de adolescentes que se encuentran casadas o en unión libre, mostraron menos niveles de depresión que el grupo de las solteras, no obstante, en este estudio los estadísticos de ANOVA evidnciarón que no existía

Tabla 6. Prueba de Bonferroni para la variable estrés.

\begin{tabular}{|c|c|c|c|c|c|c|}
\hline \multirow{2}{*}{$\begin{array}{l}\text { Condición de } \\
\text { estado civil }\end{array}$} & & \multirow{2}{*}{$\begin{array}{l}\text { Diferencia } \\
\text { de medias }\end{array}$} & \multirow{2}{*}{$\begin{array}{l}\text { Error } \\
\text { estándar }\end{array}$} & \multirow{2}{*}{$\mathrm{p}$} & \multicolumn{2}{|c|}{$95 \%$ Intervalo de confianza } \\
\hline & & & & & Límite inferior & Límite superior \\
\hline \multirow{3}{*}{$\begin{array}{l}\text { Soltera } \\
\text { viviendo con la } \\
\text { propia familia }\end{array}$} & $\begin{array}{l}\text { Casada/unida viviendo con la propia } \\
\text { familia }\end{array}$ & 4.06 & 2.41 & .576 & -2.46 & 10.58 \\
\hline & $\begin{array}{l}\text { Casada/unida viviendo con la fami- } \\
\text { lia de la pareja }\end{array}$ & $7.56^{*}$ & 2.20 & .006 & 1.62 & 13.51 \\
\hline & Pareja viviendo sola & $12.36^{*}$ & 2.80 & .000 & 4.78 & 19.95 \\
\hline \multirow{3}{*}{$\begin{array}{l}\text { Casada/unida } \\
\text { viviendo con la } \\
\text { propia familia }\end{array}$} & $\begin{array}{l}\text { Soltera viviendo con la propia } \\
\text { familia }\end{array}$ & -4.06 & 2.41 & .576 & -10.58 & 2.46 \\
\hline & $\begin{array}{l}\text { Casada/unida viviendo con la fami- } \\
\text { lia de la pareja }\end{array}$ & 3.51 & 2.55 & 1.000 & -3.40 & 10.42 \\
\hline & Pareja viviendo sola & 8.30 & 3.09 & .053 & -0.056 & 16.67 \\
\hline \multirow{3}{*}{$\begin{array}{l}\text { Casada/unida } \\
\text { viviendo con } \\
\text { la familia de la } \\
\text { pareja }\end{array}$} & $\begin{array}{l}\text { Soltera viviendo con la propia } \\
\text { familia }\end{array}$ & $-7.56^{*}$ & 2.20 & .006 & -13.51 & -1.62 \\
\hline & $\begin{array}{l}\text { Casada/unida viviendo con la propia } \\
\text { familia }\end{array}$ & -3.51 & 2.55 & 1.000 & -10.42 & 3.40 \\
\hline & Pareja viviendo sola & 4.79 & 2.96 & .630 & -3.12 & 12.72 \\
\hline \multirow{3}{*}{ Pareja sola } & $\begin{array}{l}\text { Soltera viviendo con la propia } \\
\text { familia }\end{array}$ & $-12.36^{*}$ & 2.80 & .000 & -19.95 & -4.78 \\
\hline & $\begin{array}{l}\text { Casada/unida viviendo con la propia } \\
\text { familia }\end{array}$ & -8.30 & 3.09 & .053 & -16.67 & 0.056 \\
\hline & $\begin{array}{l}\text { Casada/unida viviendo con la fami- } \\
\text { lia de la pareja }\end{array}$ & -4.80 & 2.93 & .630 & -12.72 & 3.12 \\
\hline
\end{tabular}

$* \mathrm{p}<.05$ 
una diferencia significativa entre los grupos de cada condición de estado civil. Las medias de depresión son muy cercanas unas a otras y cabe destacar que las adolescentes solteras que viven con la propia familia y las casadas/unidas que viven con la propia familia son quienes presentan las medias más altas de los puntajes; le siguen las casadas/unidas viviendo con la familia de la pareja, hasta llegar a la condición de estado civil con menor nivel de depresión, siendo la pareja sola (casadas/unidas que viven de manera independiente de ambos senos familiares), sin llegar estas diferencias de medias a un nivel estadísticamente significativo.

En cuanto a los niveles de estrés, las cuatro condiciones de estado civil mostraron niveles entre altos y moderados, con diferencias significativas entre sus medias. El grupo más estresado correspondió a las solteras que viven con la propia familia y el menos estresado al de la pareja sola. Las condiciones de estado civil con diferencia significativa entre sus medias de acuerdo a los estadísticos de ANOVA, correspondieron a las solteras viviendo con la propia familia en relación a las casadas/unidas viviendo con la familia de la pareja ( $\mathrm{p}=$ .006) y a la pareja sola $(\mathrm{p}=.001)$. Lo anterior indica que las casadas/unidas viviendo con la propia familia no presentaron diferencia significativa entre sus medias de estrés hacia los otros grupos.

La escala de estrés percibido ha sido un instrumento utilizado en numerosos estudios, los cuales sirven de referencia para establecer una comparación con la muestra de adolescentes embarazadas de esta investigación. Tal es así, que la media de estrés para este grupo de adolescentes $($ Media $=26.37$, D.E. $=8.95)$ es cercana a la entregada por Pedrero-Pérez y OlivarArroyo (2010) en sujetos adictos a sustancias (Media $=27.9$, D.E. $=10.6)$ y en pacientes con cardiopatía isquémica $($ Media $=27.9$; D.E. $=8.9)$, cifras reportadas por Canales (2009).

Sin embargo, cabe destacar que la media de estrés percibido de esta muestra con adolescentes embarazadas es mayor a la de personas con VIH (Media = 22.6; D.E. $=8.67$, Remor y Carrobles, 2001), epidermollisis bullosa - enfermedad dermatológica $-($ Media $=21.7$; D.E. $=6.54$, Reyna, 2009) y en estudiantes universitarias $($ Media $=21.0$; D.E. $=4.1$, González \& Landero, 2008).

En el caso de los estresores, no se encontraron diferencias significativas en las medias de la variable en cuestión, esto se debe principalmente a que la mayoría de los eventos contenidos en el Cuestionario de Sucesos Vitales, no han sido enfrentados por las adolescentes de la muestra, no obstante, se puede destacar que en congruencia con Ramsey y otros (1986), las situaciones sociales fueron las que mayormente se reportaron como estresores en las cuatro condiciones de estado civil, siendo las solteras que viven con la propia familia las que encabezan esta lista, seguidas por las casadas/ unidas viviendo con la familia de la pareja, casadas/ unidas viviendo con la propia familia y la pareja sola.

Retomando lo anterior, Della (2006) argumenta que el embarazo en las adolescentes resulta ser un evento generador de estrés (estresor), hecho que es sustentado por este estudio ya que siendo el único ítem para la sub-escala de hijos en el CSV, la mayoría (64\%) de las adolescentes solteras viviendo con la propia familia consideraron el quedar embarazada como un hecho negativo y en un menor porcentaje, las casadas/ unidas viviendo con la propia familia con el $23 \%$; las condiciones de estado civil de pareja sola con $18 \%$ y casadas/unidas viviendo con la familia de la pareja con $17 \%$ mostraron poseer los porcentajes más bajos.

El hecho de que únicamente el estrés haya presentado diferencia significativa entre las medias de las cuatro condiciones de estado civil, puede deberse a que aunque las adolescentes se enfrenten a situaciones similares (sucesos vitales), la percepción de estas varía en cada individuo, por lo que la percepción del estrés es distinta. Lo mismo ocurre con la depresión.

En conclusión y de acuerdo a lo expuesto por Ramos y otros (2009), las mujeres embarazadas deprimidas y aún más las adolescentes, pueden adoptar conductas de riesgo como fumar, ingerir bebidas alcohólicas, menor control prenatal, aborto espontáneo, parto prematuro, retardo en el crecimiento intrauterino, bajo peso y talla al nacer del producto, hipertensión arterial en la madre, además de un riesgo aumentado de padecer depresión postparto (Da Costa \& otros, 2000; Rahman, Iqbal \& Harrington, 2003). Dicho esto, podemos concluir que las adolescentes primigestas que viven con la propia familia y especialmente las solteras, pueden estar predispuestas en mayor medida a presentar estos problemas en relación a las demás condiciones de estado civil, además de tener una posibilidad más alta o de padecer depresión en el postparto. En consecuencias, se hace la invitación a considerar de una manera más atenta y prioritaria a este grupo de adolescentes en los servicios psicológicos y médicos a los que acudan.

\section{Referencias}

Alderdice, F. y Lynn, F. (2009). Stress in pregnancy: identifying and supporting women. British Journal of Midwifery, 17(9), 552-559. Bartolomucci, A., y Leopardi, R. (2009). Stress and Depression: Preclinical Research and Clinical Implications. PLoS ONE, 4(1), 1-5. 
Beck, A. T., Ward, C. H., Mendelson, M., Mock, J. y Erbaugh, J. (1961). An inventory for measuring depression. Archive of General Psychiatry, 4, 53-61.

Billingham, K. (2011). Preparing for parenthood: The role of antenatal education: The journal of the health visitors' association. Community Practitioner, 84(5), 36-38.

Canales, L. (2009). Intervención cognitivo-conductual para la disminución del estrés en pacientes con cardiopatía isquémica. Tesis de Maestría. Facultad de Psicología. UANL.

Cohen, S., Kamarak, T. y Merlmelstein, R. (1983). A global measure of perceived stress. Journal of Health and Social Behavior, 24, 385-396.

Cohen, S. y Williamson, G. M. (1988). Perceived stress in a probability sample of the United States. En S. Spacapan y S. Oskamp (Eds.), The social psychology of health (pp. 31-67). Beverly Hills, CA: Sage.

Conde-Agudelo, A., Belizan, J.M. y Lammers, C. (2005). Maternalperinatal morbidity and mortality associated with adolescent pregnancy in Latin America: Cross sectional study. American Journal of Obstetrics and Gynecology, 192, 342-349.

Da Costa, D., Dritsa, M., Larouche, J. y Brender, W. (2000). Psychosocial predictors of labor/delivery complications and infant birth weight: a prospective multivariate study. Journal of Psychosomatic Obstetrics and Gynaecology, 21, 137-148.

Deal, L. y Holt, V. (1988). Young Maternal Age and Depressive Symptoms: Results from the 1988 National Maternal and Infant Health Survey, American Journal of Public Health, 88(2), 266.

Della, M. (2006). Estrategias de afrontamiento (coping) en adolescentes embarazadas escolarizadas. Revista Iberoamericana de Educación, 38(3), 1-14.

Díaz, V. (2003). Embarazo en la adolescencia. Gaceta Médica de México, 139(1), 1-10.

Díaz-Franco, E. (2007). Guía clínica de intervención psicológica del embarazo en la adolescencia. Perinatología y Reproducción Humana, 21(2), $100-110$.

Figueiredo, B., Bifulco, A., Pacheco, A., Costa, R. y Magarinho, R. (2006). Teenage pregnancy, attachment style, and depression: A comparison of teenage and adult pregnant women in a Portuguese series. Attachment \& Human Development, 8(2), 123-138.

Gómez, M.E. y Aldana, E. (2007). Alteraciones psicológicas en la mujer con embarazo de alto riesgo. Psicología y Salud, 17(1), 53-61.

González, M.T. y Landero, R. (2007). Factor Structure of the Perceived Stress Scale (PSS) in a sample from Mexico. The Spanish Journal of Psychology, 10(1), 199-206.

González, M. T. y Landero, R. (2007a). Escala de cansancio emocional (ECE) para estudiantes universitarios: propiedades psicométricas en una muestra de México. Anales de Psicología, 23(2), 253-257.

González, M.T. y Landero, R. (2008). Confirmación de un modelo explicativo del estrés y de los síntomas psicosomáticos mediante ecuaciones estructurales. Revista Panamericana de Salud Pública, 23 (1), 7-18.

González, M.T., García-Campayo, J. y Landero, R. (2011). The role of stress transactional theory on the development of fibromyalgia: A structural equation model. Actas españolas de psiquiatría, en prensa.

Grote, N.K. y Bledsoe, S.E. (2007). Predicting Postpartum Depressive Symptoms in New Mothers: The Role of Optimism and Stress Frequency during Pregnancy. Health \& Social Work, 32(2), 107118.

Joshi, T.P. (1998) Affective disorders in children and adolescents. En H.S. Guhman y R.M. Sarles (Eds.), Handbook of child and adolescent outpatient, day treatment and community psyquiatry. Castleton, N.Y.: Hamilton Printing.
Kovacs, M. (2006). Next steps for research on child and adolescent depression prevention. American Journal of Preventive Medicine, 31, 184-185.

Lanzi, R., Bert, S. y Jacobs, B. (2009). Depression among a sample of first-time adolescent and adult mothers. Journal of Child and Adolescent Psychiatric Nursing, 22(4), 194-202.

Latendresse, G. (2009). The interaction between chronic stress and pregnancy: Preterm birth from a biobehavioral perspective. Journal of Midwifery \& Women's Health, 54(1), 8-17.

Lazarus, R. (2000). Estrés y emoción. Manejo e implicaciones en nuestra salud. España: Desclée de Brouwer.

Moral, J. (2011). Propiedades psicométricas de un formato de aplicación simplificado para el inventario de depresión de Beck. Revista Mexicana de Investigación en Psicología Social y de la Salud, 1(2). 4-18.

Moral, J., González, M.T. y Landero, R. (en prensa). Tratamiento de la fibromialgia en mujeres y evaluación del burnout en amas de casa. México: Plaza y Valdez.

Moral, J. y Martínez, J. (2009). Reacción ante el diagnóstico de cáncer en un hijo: estrés y afrontamiento. Psicología y Salud, 19(2), 189-196.

Oviedo, H., Lira, J., Ito, A. y Grosso, J.M. (2007). Causas de nacimiento pretérmino entre madres adolescentes. Ginecología y Obstetricia de México, 75, 17-23.

Pedrero, E. y Olivar, A. (2010). Estrés percibido en adictos a sustancias en tratamiento mediante la escala de Cohen: propiedades psicométricas y resultados de su aplicación. Anales de psicología, 26 (2), 302-309. Consultado el 5 de febrero del 2010. Disponible en: http://www.um.es/analesps/v26/v26_2/14-26_2.pdf

Ramos, R.Y., Barriga J.A. y Pérez, J. (2009). Embarazo en adolescentes como factor de riesgo para maltrato fetal. Ginecología y Obstetricia de México, 77(7) 311-316.

Ramsey, C.N., Abell, T.D. y Baker, L.C. (1986). The relationship between family functioning, life events, family structure, and the outcome of pregnancy. Journal of Family Practice, 22, 521-527.

Remor, E. y Carrobles, J. (2001). Versión Española de la escala de estrés percibido (PSS-14): Estudio psicométrico en una muestra VIH+. Ansiedad y Estrés, 7 (2-3), 195-201.

Reyna, A. (2009). Psicodermatología: aspectos psicológicos en personas con epidermolisis bullosa. Tesis de Maestría. Facultad de Psicología. UANL.

Sánchez, E., Garrido, A. y Álvaro, J.L. (2003). Un modelo psicosociológico para el estudio de la salud mental. Psicología Social, 18(1), 17-33.

Sandín, B. y Chorot, P. (1987). Cuestionario de Sucesos Vitales $(C S V)$. Madrid: Universidad Nacional de Educación a Distancia (UNED).

Sepúlveda, J., Bustreo, F., Tapia, R., Rivera, J., Lozano, R., Oláiz, G., Partida, V., García-García, L. y Valdespino, J.L. (2006). Improvement of child survival in Mexico: the diagnostical approach. Lancet, 368, 2017-2027. Consultado el 27 de marzo del 2010. Disponible en: http://www.thelancet.com/search/results?searchT erm $=$ Sepulveda\&fieldName $=$ Authors $\&$ journalFromWhichSea rchStarted $=$

Vázquez, C. y Sanz, J. (1997). Fiabilidad y valores normativos de la versión española del inventario para la depresión de Beck de 1978. Clínica y Salud, 8(3), 403-422.

Zelkowitz, P., Schinazi, J., Katofsky, L., Saucier, J.F., Valenzuela, M., Westreich, R. y Dayan, J. (2004). Factors Associated with Depression in Pregnant Immigrant Women. Trascultural Psychiatry, 41(4), 445-464.

Zuckerman, B., Bauchner, H., Parker, S. y Cabral, H. (1990). Maternal depressive symptoms during pregnancy and newborn irritability. Journal of Developmental \& Behavioural Pediatrics, 11, 190-194. 